\title{
Refractory neuro-Sweet disease successfully treated with tocilizumab and mycophenolate mofetil
}

\author{
Sungeun Hwang ${ }^{1,2}$, Hyoshin Son ${ }^{1,2}$, Jangsup Moon ${ }^{1,2,3}$, Soon-Tae Lee ${ }^{1,2}$, Keun Hwa Jung ${ }^{1,2}$, Kyung-ll Park ${ }^{1,2,4}$, \\ Sang Kun Lee ${ }^{1,2}$, Kon Chu ${ }^{1,2}$ \\ ${ }^{1}$ Department of Neurology, Seoul National University Hospital, Seoul, Korea \\ ${ }^{2}$ Laboratory for Neurotherapeutics, Center for Medical Innovations, Biomedical Research Institute, Seoul National University Hospital, \\ Seoul, Korea \\ ${ }^{3}$ Rare Disease Center, Seoul National University Hospital, Seoul, Korea \\ ${ }^{4}$ Department of Neurology, Seoul National University Hospital Healthcare System Gangnam Center, Seoul, Korea
}

\begin{abstract}
Sweet syndrome, or acute febrile neutrophilic dermatosis, is mainly a dermatologic condition presenting with erythematous plaques; however, neutrophils infiltrate multiple systems. Neuro-Sweet disease is a neurological manifestation of Sweet syndrome and a rare cause of recurrent aseptic meningoencephalitis, which needs to be distinguished from neuro-Behçet disease. Although neuro-Sweet disease generally responds well to corticosteroids, relapsing neuro-Sweet disease is not an exceptional case. Herein, we present a case of a 51-year-old male with recurrent encephalitis followed by erythematous plaques. The patient was confirmed as Sweet syndrome based on skin biopsy and showed partial response to corticosteroids. With intravenous immunoglobulin, rituximab, tocilizumab, and mycophenolate mofetil, his neurologic symptoms were fully recovered.
\end{abstract}

Keywords: Sweet syndrome, Tocilizumab, Mycophenolic acid

\section{Introduction}

Sweet syndrome (acute febrile neutrophilic dermatosis) was first described by Dr. Robert Douglas Sweet in 1964 [1]. The syndrome is characterized by fever, neutrophilic leukocytosis, and tender erythematous plaques. Biopsy of erythematous plaques shows a dense dermal infiltration with mature neutrophils. Response to corticosteroid treatment is generally rapid and favorable. Neurological manifestation of Sweet syndrome, termed neuro-Sweet disease, is a rare cause of aseptic meningoencephalitis. Neuro-Sweet disease also responds to corticosteroids, however, refractory cases with multiple relapses have occurred. Herein, we report a case of a patient with refractory neuro-Sweet disease, who was successfully treated with intravenous immunoglobulin (IVIG), rituximab, tocilizumab, and mycophenolate mofetil.

\section{Case Report}

A 51-year-old male with a history of recurrent episodes of unconsciousness (10, 7, 2 years, and 4 months before the current visit) presented with cognitive impairment. The patient was admitted to another hospital for altered mental state 10 and 7 years before the current visit. Although no definitive diagnosis was made, his consciousness was restored with supportive treatment. When visiting another hospital 2 years before his current visit (May 2016), the patient had erythematous plaques on the neck and legs accompanied by headache, myalgia, febrile sense, and unconsciousness. On admission to our hospital, a brain magnetic resonance imaging (MRI) scan showed T2 hyperintensities on bilateral basal ganglia, frontal, temporal, and insular subcortices (Figure 1A and B). Biopsy of the posterior neck skin lesion on admission revealed dermal neutrophilic infiltration

Received: November 16, 2020 Revised: December 1, 2020 Accepted: December 1, 2020

Correspondence: Kon Chu

Department of Neurology, Seoul National University Hospital, 101 Daehak-ro, Jongno-gu, Seoul 03080, Korea

E-mail: stemcell.snu@gmail.com

ORCID: https://orcid.org/0000-0001-5863-0302

Copyright (C) 2021 by The Korean Encephalitis and Neuroinflammation Society

This is an open access article distributed under the terms of the Creative Commons Attribution Non-Commercial License (http://creativecommons.org/licenses/by-nc/4.0/) which permits unrestricted non-commercial use, distribution, and reproduction in any medium, provided the original work is properly cited. 
compatible with Sweet syndrome. The patient received symptomatic treatments and erythematous plaques were resolved without corticosteroid therapy. Brain MRI after 2 months showed resolution of bilateral basal ganglia and subcortical T2 hyperintense lesions (Figure $1 \mathrm{C}$ and D).

The patient presented 4 months earlier (January 2018) with sore throat, headache, myalgia, febrile sense, unconsciousness, and erythematous nodules on his face spreading to the whole body. T2 hyperintense lesions were observed in the left thalamus, midbrain, and pons on brain MRI scan (Figure $1 \mathrm{E}$ and F). After treatment with high-dose corticosteroids for 5 days, his symptoms improved and T2 hyperintensities on brain MRI scan disappeared (Figure $1 \mathrm{G}$ and $\mathrm{H}$ ). However, he complained of persistent dysarthria and cognitive impairment for months.

The subject was admitted to our center for further treatment in May 2018. Neurologic examination revealed mild dysarthria without other cranial nerve dysfunction. Fever and erythematous plaques were absent on admission. Neutrophilia (leukocytes, $14,880 / \mu \mathrm{L}$; neutrophils, $11,859 / \mu \mathrm{L} ; 79.7 \%$ of neutrophils) and elevated C-reactive protein (CRP) level (1.17 mg/dL) were observed despite oral prednisolone maintenance (10 mg/day) for the preceding 4 months. Cerebrospinal fluid examination was unremarkable. Viral, fungal, tuberculous, or bacterial pathogens were not detected. Human leukocyte antigen (HLA) typing confirmed HLA-B52/B54. Mini-mental state examination score was 25 of 30 with impaired memory registration, recall, and calculation. Frontal assessment battery showed decreased word fluency. Clinical assessment scale for autoimmune encephalitis (CASE) score was 3 and modified Rankin scale (mRS) score was 2 [2].

IVIG treatment ( $400 \mathrm{mg} / \mathrm{kg} /$ day for 5 days) was initiated followed by weekly rituximab ( $375 \mathrm{mg} / \mathrm{m}^{2}$ for 4 weeks). After the 2nd administration of rituximab, dysarthria and language fluency were slightly improved (CASE score, 2; mRS score, 2). After the 4 th dose of rituximab, brain MRI scan showed new T2 hyperintensities on bilateral frontal, temporal, and insular subcortices (Figure 1I and J). However, neurologic deterioration was not observed and four cycles of monthly rituximab therapy were

Figure 1 Serial MRI scan images (fluid attenuated inversion recovery images)
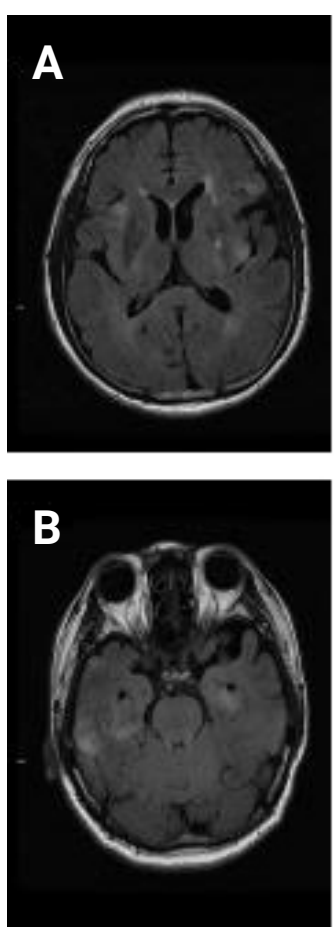
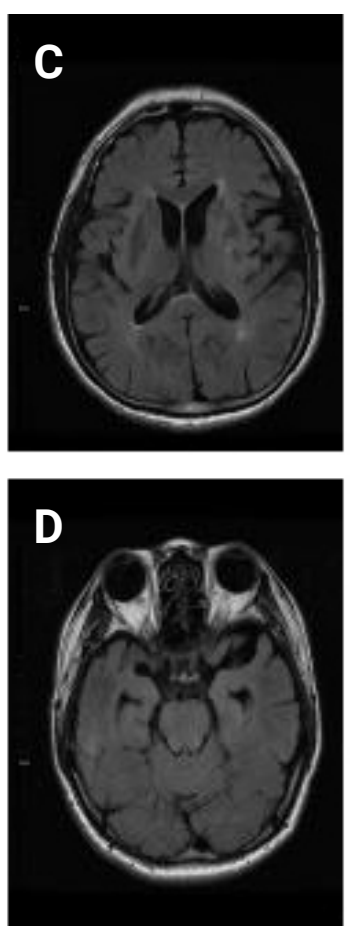
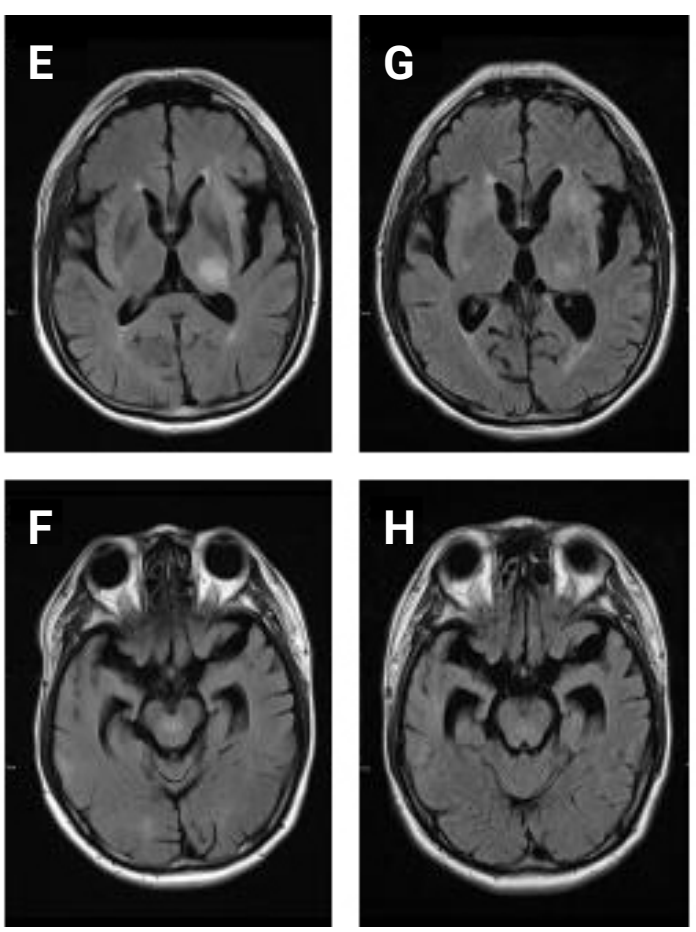

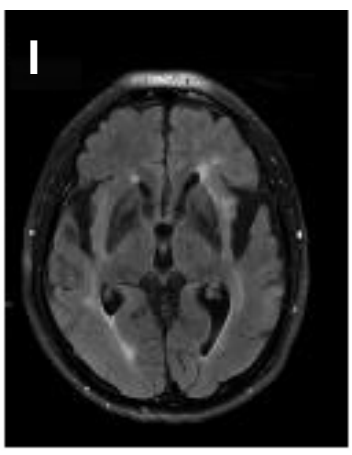

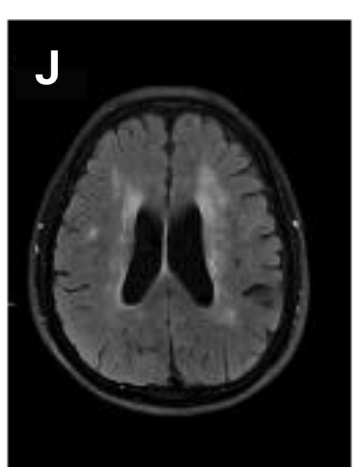

MRI scan in May 2016 (A, B) shows T2 hyperintense lesions in bilateral basal ganglia and subcortical frontal, temporal, and insular lobes, which were reduced on follow-up MRI scan in July 2016 (C, D). (E, F) New T2 hyperintense lesions in left thalamus, midbrain, and pons were observed in February 2018. (G, H) Partial resolution of previous lesions was observed in March 2018. (I, J) In June 2018, MRI scan revealed bilateral subcortical T2 hyperintensities in bilateral frontal, temporal, insular, and parietal lobes. These lesions remained on follow-up MRIs in December 2018 and May 2019 (not shown).

MRI, magnetic resonance imaging. 
maintained. Dysarthria or language dysfunction was not observed but mild memory dysfunction remained (CASE score, 1; mRS score, 1).

In November 2018, an erythematous rash developed without neurologic deterioration. Oral mycophenolate mofetil (500 mg/ day) and high-dose corticosteroid were initiated resulting in resolution of erythematous plaques. Intravenous tocilizumab (6 $\mathrm{mg} / \mathrm{kg}$ every month) was administered from December 2018 to June 2019. Erythematous plaques recurred in February and May 2019 (Figure 2A), which resolved with tocilizumab (and combined high-dose intravenous corticosteroid in February 2019). Skin biopsy from a plaque on the posterior neck in May 2019 (Figure 2B) revealed dermal neutrophilic infiltration suggestive of Sweet syndrome. After the 6th injection of tocilizumab, only mycophenolate mofetil was maintained without recurrence of rash and neurologic symptoms. Mycophenolate mofetil was discontinued in December 2019. Rash and neurologic symptoms

Figure 2 Posterior neck plaque in May 2019
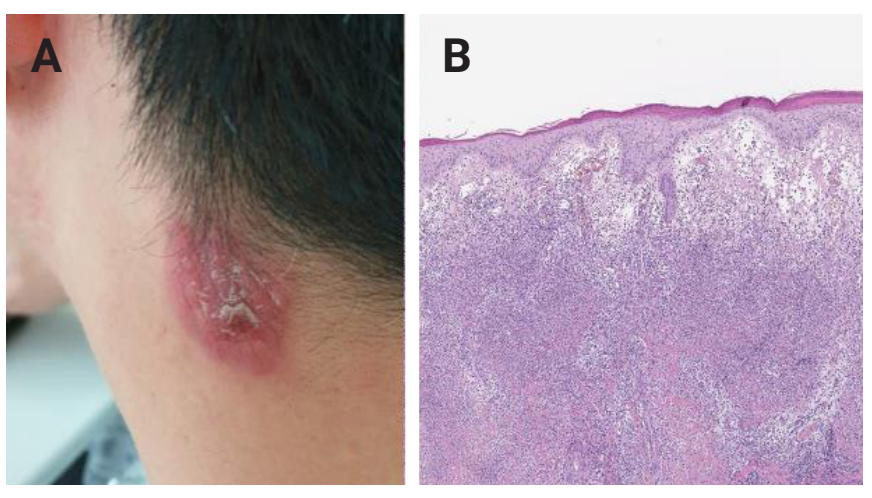

(A) Erythematous plaque on left posterior neck. (B) Skin biopsy of erythematous plaque on left posterior neck (H\&E stain, $\times 40)$. Mixed lymphohistiocytic and numerous neutrophilic infiltrations in dermis. did not relapse until the last visit in March 2020 (CASE score, 0; mRS score, 0 ). Time course of immunotherapies and clinical events are depicted in Figure 3.

Written informed consent was obtained for publication of this case report and accompanying images.

\section{Discussion}

Sweet syndrome (acute febrile neutrophilic dermatosis) is characterized by fever, leukocytosis, and tender erythematous skin lesions [1]. Erythema typically involves head, neck, and limbs. Leukocytosis (neutrophilia) and elevated CRP and erythrocyte sedimentation rate are common laboratory findings. Skin biopsy shows dermal neutrophilic infiltration in the absence of vasculitis. Although skin is most commonly involved, other organs such as eyes, liver, kidneys, and lungs can be infiltrated with neutrophils [3]. Response to corticosteroid is favorable, resulting in complete remission without scarring in most cases [4].

Neurologic manifestation of Sweet syndrome, termed neuro-Sweet disease, has been reported since the 1990s [5]. Common presentation of neuro-Sweet disease is aseptic meningoencephalitis with or without erythematous plaques [3]. Various symptoms such as altered consciousness, headache, cognitive impairment, aphasia, or peripheral neuropathy can be observed. Typically, erythematous plaques precede neurologic symptoms; however, cases of encephalitis followed by skin involvement or cases without erythematous plaques have been reported [6]. Computed tomography or MRI scans reveal multiple central nervous system lesions [3]. Basal ganglia and brainstem are frequently involved similar to neuro-Behçet disease. Because skin is commonly involved in neuro-Sweet disease and neuro-Behçet disease, differentiating these two conditions is important. In neuro-Behçet disease, skin biopsy shows vasculi-

Figure 3 Time course of immunotherapies and clinical events

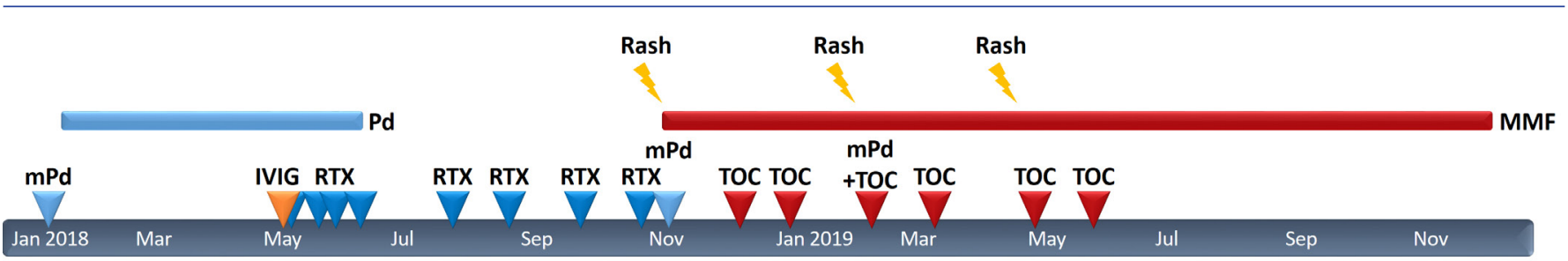

Arrows and bars on the timelines indicate the timing/duration of each immunotherapy. Yellow lightning indicates timing of relapsed erythematous plaques.

mPd, intravenous high-dose methylprednisolone; Pd, oral prednisolone; IVIG, intravenous immunoglobulin; RTX, rituximab; TOC, tocilizumab; MMF, mycophenolate mofetil. 
tis in contrast to dermal neutrophilic infiltration in neuro-Sweet disease. Neuro-Sweet disease usually responds to corticosteroids, and in some cases supportive treatment can lead to spontaneous remission. HLA-B54 (63\% in neuro-Sweet disease vs. $14 \%$ in Japanese population) and HLA-Cw (89\% in neuro-Sweet disease vs. $28 \%$ in Japanese population) are frequently detected in patients with neuro-Sweet disease [3]. Although recurrence is common, there is no established preventive therapy to date. Conversely, response to corticosteroids is poor and HLA-B51 is frequently detected in patients with neuro-Behçet disease [7].

Treatment options other than corticosteroids include colchicine and potassium iodide as first-line treatment options for dermal involvement of Sweet syndrome [8]. Nonsteroidal anti-inflammatory drugs, dapsone, clofazimine, cyclosporine, and thalidomide are second-line therapies. There are reports of effective treatment of Sweet syndrome with methotrexate, interferon- $\alpha$, IVIG, anakinra, or anti-tumor necrosis factor (TNF)- $\alpha$ [8,9]. Rituximab (anti-CD20 monoclonal antibody) and tocilizumab (anti-interleukin-6 monoclonal antibody) have shown efficacy in case reports of refractory Sweet syndrome [10,11]. However, possible anti-TNF- $\alpha$ - or tocilizumab-induced Sweet syndrome has been reported, and the effectiveness of these drugs remains controversial [12].

In the present case, the patient had a history of recurrent neuro-Sweet syndrome. Because skin was involved years after the initial neurologic manifestation, he experienced a diagnostic odyssey. He was undiagnosed until the erythematous plaques appeared 2 years before the current visit, which prompted skin biopsy confirming the diagnosis of neuro-Sweet disease. Despite spontaneous remission after supportive treatment 10, 7 , and 2 years earlier, high-dose corticosteroid treatment induced partial remission 4 months before the current visit. Dysarthria and cognitive impairment remained even with long-term maintenance of oral corticosteroid. Additional immunotherapy with IVIG, rituximab, mycophenolate mofetil, and tocilizumab induced complete remission of neuro-Sweet disease without neurologic sequela.

The present case demonstrates efficacy of multiple immunotherapies in neuro-Sweet disease. Although numerous reports of dermal manifestation of Sweet syndrome treated with immunomodulatory drugs exist, reports on immunotherapy treatment in neuro-Sweet disease are limited. Reportedly, cyclosporine, dapsone, and infliximab (TNF- $\alpha$ inhibitor) have been used in cases of corticosteroid-resistant or corticosteroid-dependent neuro-Sweet disease $[13,14]$. To the best of our knowledge, this is the first case of neuro-Sweet disease treated with rituximab, tocilizumab, and mycophenolate mofetil. Tocilizumab, which is commonly used in rheumatoid arthritis, is reportedly associated with neutropenia in some cases [15]. Although the exact mechanism is not established, this anti-neutrophil effect may serve as a therapeutic target in Sweet syndrome [16]. A case of Sweet syndrome or neuro-Sweet disease treated with mycophenolate mofetil has not been reported to date. Although mycophenolate mofetil was initiated after cutaneous relapse in November 2018, the contribution of mycophenolate mofetil cannot be established due to concomitant administration of corticosteroids followed by tocilizumab. However, tentative therapeutic effects of mycophenolate mofetil should be further studied in refractory cases of neuro-Sweet disease. Reversibility of neurologic symptoms even after months from relapse highlights the importance of adequate immunotherapy in steroid-unresponsive neuro-Sweet disease. The mechanism by which each immune-modulating agent acts on neuro-Sweet disease, and priority of each immunotherapy, remain to be elucidated.

\section{Conflicts of Interest}

Jangsup Moon, Soon-Tae Lee, Kyung-Il Park, Sang Kun Lee, Kon Chu have been editorial board of encephalitis since October 2020. They were not involved in the review process of this case report. No other potential conflict of interest relevant to this article was reported.

\section{Author Contributions}

Conceptualization: S Hwang, K Chu; Data curation, Investigation: S Hwang, H Son; Formal analysis, Visualization: S Hwang; Methodology: S Hwang, J Moon, K Chu; Project administration, Resources, Supervision: K Chu; Writing-original draft: S Hwang; Writing-review and editing: all authors.

\section{References}

1. Sweet RD. An acute febrile neutrophilic dermatosis. Br J Dermatol 1964;76:349-356.

2. Lim JA, Lee ST, Moon J, et al. Development of the clinical assessment scale in autoimmune encephalitis. Ann Neurol 2019;85:352-358.

3. Hisanaga K, Iwasaki Y, Itoyama Y; Neuro-Sweet Disease Study Group. Neuro-Sweet disease: clinical manifestations and criteria for diagnosis. Neurology 2005;64:1756-1761. 
4. von den Driesch P. Sweet's syndrome (acute febrile neutrophilic dermatosis). J Am Acad Dermatol 1994;31:535-556.

5. Hisanaga K, Hosokawa M, Sato N, Mochizuki H, Itoyama Y, Iwasaki Y. "Neuro-sweet disease": benign recurrent encephalitis with neutrophilic dermatosis. Arch Neurol 1999;56:1010-1013.

6. Cohen PR. Sweet's syndrome: a comprehensive review of an acute febrile neutrophilic dermatosis. Orphanet J Rare Dis 2007;2:34.

7. Leccese P, Alpsoy E. Behçet's disease: an overview of etiopathogenesis. Front Immunol 2019;10:1067.

8. Schadt CR, Callen JP. Management of neutrophilic dermatoses. Dermatol Ther 2012;25:158-172.

9. Kluger N, Gil-Bistes D, Guillot B, Bessis D. Efficacy of anti-interleukin-1 receptor antagonist anakinra (Kineret ${ }^{\circ}$ ) in a case of refractory Sweet's syndrome. Dermatology 2011;222:123-127.

10. Seminario-Vidal L, Guerrero C, Sami N. Refractory Sweet's syndrome successfully treated with rituximab. JAAD Case Rep 2015;1:123-125.

11. Cook QS, Zdanski CJ, Burkhart CN, Googe PB, Thompson P, Wu EY. Idiopathic, refractory Sweet's syndrome associated with common variable immunodeficiency: a case report and literature review. Curr Allergy Asthma Rep 2019;19:32.

12. Filippi F, Chessa MA, Patrizi A, Baraldi C, Ferrara F, Bardazzi F. Tocilizumab-induced Sweet syndrome in a patient with polymyalgia rheumatica. Dermatol Pract Concept 2019;10:e2020019.

13. Drago F, Ciccarese G, Agnoletti AF, Sarocchi F, Parodi A. Neuro sweet syndrome: a systematic review: a rare complication of Sweet syndrome. Acta Neurol Belg 2017;117:33-42.

14. Lobo AM, Stacy R, Cestari D, Stone JH, Jakobiec FA, Sobrin L. Optic nerve involvement with panuveitis in Sweet syndrome. Ocul Immunol Inflamm 2011;19:167-170.

15. Nakamura I, Omata Y, Naito M, Ito K. Blockade of interleukin 6 signaling induces marked neutropenia in patients with rheumatoid arthritis. J Rheumatol 2009;36:459-460.

16. Wright HL, Cross AL, Edwards SW, Moots RJ. Effects of IL-6 and IL-6 blockade on neutrophil function in vitro and in vivo. Rheumatology (Oxford) 2014;53:1321-1331. 